\title{
Collaborative caching priority for processing requests in MANETs
}

\begin{abstract}
Accessing distant sources in MANETs leads to poor performance and sometimes impossible due to regular disconnection of mobile hosts. Several approaches have been proposed to improve data accessibility and reduce delay in serving requests. These approaches adopted the collaborative caching techniques, enabling various mobile hosts to cache and share data items in their local caches. However, processing requests based on their classification have not been tackled in previous works to reduce the average delay. In this paper, we propose a collaborative caching priority approach, which serves requests based on their classifications either priority or normal. This is to ensure that priority requests are served with minimum cache discovery overhead and with less delay in fetching data items that are cached in MANETs. The experimental results show that the proposed approach improved the performance of collaborative caching and outperformed the cooperative and adaptive system (COACS), with a decrement of $30.42 \%$ in average delay and an increment of $21.26 \%$ in hit ratio.
\end{abstract}

Keyword: Cache management; Collaborative caching; MANETs; Database requests 